\title{
Production of free methylarginines via the proteasome and autophagy pathways in cultured cells
}

\author{
TAKUMA SHIRAKAWA ${ }^{1}$, KOICHIRO KAKO ${ }^{1}$, TAKASHI SHIMADA ${ }^{1}$, YUSUKE NAGASHIMA ${ }^{1}$, \\ AYUMI NAKAMURA ${ }^{1}$, JUNJI ISHIDA ${ }^{1,2}$ and AKIYOSHI FUKAMIZU ${ }^{1,2}$ \\ ${ }^{1}$ Graduate School of Life and Environmental Sciences; ${ }^{2}$ Life Science Center, Tsukuba Advanced \\ Research Alliance (TARA), University of Tsukuba, Tsukuba, Ibaraki 305-8577, Japan
}

Received December 3, 2010; Accepted April 8, 2011

DOI: $10.3892 / \mathrm{mmr} .2011 .488$

\begin{abstract}
N^{\mathrm{G}}$-monomethylarginine (MMA) and asymmetric $\omega-N^{\mathrm{G}}, \omega-N^{\mathrm{G}}$-dimethylarginine (ADMA), are endogenous competitive inhibitors for three isoforms of nitric oxide synthase (NOS). Although free methylarginines are thought to be liberated through the intracellular proteolysis of proteins methylated by protein arginine methyltransferases (PRMTs), the degradation pathways of the arginine-methylated proteins involved in the biosynthesis of free methylarginines have yet to be determined. In this study, the biosynthesis of free methylarginines with cultured cells was analyzed as follows: first, we established a method for quantifying trace amounts of free intracellular methylarginines by means of ultra high-performance liquid chromatography (UPLC). Second, we determined the type of methylation produced in the cultured cell lines using matrix-assisted laser desorption/ionization quadrupole ion trap time-of-flight tandem mass spectrometry (MALDI-QIT-TOF/ MS). Finally, we investigated whether methylarginines are generated via the proteasome and autophagy pathways, the primary intracellular protein degradation systems. By using specific inhibitors for each pathway, we found that the blockade of proteasome activity reduced the amount of free ADMA and symmetric $\omega-N^{\mathrm{G}}, \omega-N^{1 \mathrm{G}}$-dimethylarginine (SDMA), while the inhibition of autophagy significantly reduced cellular ADMA only. These results suggest that both the proteasome and autophagy pathways play an essential role in the production of free methylarginines.
\end{abstract}

\section{Introduction}

Protein arginine methylation has recently been established as an important post-translational modification involved in the

Correspondence to: Professor Akiyoshi Fukamizu, Life Science Center, TARA, University of Tsukuba, Tsukuba, Ibaraki 305-8577, Japan

E-mail: akif@tara.tsukuba.ac.jp

Key words: protein arginine methyltransferase (PRMT), methylarginine, proteasome, autophagy, matrix-assisted laser desorption/ionization quadrupole ion trap time-of-flight tandem mass spectrometry, ultra high-performance liquid chromatography regulation of transcriptional control, RNA processing, DNA repair and signal transduction. This modification is catalyzed by protein arginine methyltransferases (PRMTs) via the methylation of nitrogen atoms on the guanidinium side chains of arginine residues within proteins with $S$-adenosylmethionine as a methyl-donor $(1,2)$. PRMTs are classified into two main categories: type I enzymes, which catalyze the formation of $\omega-N^{\mathrm{G}}$-monomethylarginine (MMA) and asymmetric $\omega-N^{\mathrm{G}}, \omega-N^{\mathrm{G}}$-dimethylarginine (ADMA) (3-9), and type II enzymes, which generate MMA and symmetric $\omega-N^{\mathrm{G}}, \omega-N^{\mathrm{GG}}$ dimethylarginine (SDMA) (10-12).

These methylarginine derivatives are believed to be released into the cytoplasm following proteolysis, since no direct methylation reaction of arginine as a solitary amino acid has been observed (13). Among the derivatives, MMA and ADMA, but not SDMA, are endogenous inhibitors of nitric oxide (NO) synthase (NOS) through competition with free L-arginine residue (L-Arg), a specific substrate for NOS $(14,15)$. In the vasculature, NO is produced by constitutively expressed endothelial-specific NOS (eNOS). This endothelial-derived NO diffuses into the smooth muscle cell layer and activates soluble guanylate cyclase followed by smooth muscle relaxation (13). While it is clear that increased plasma concentrations of ADMA are correlated with various cardiovascular pathologies (15), and that the administration of ADMA has significant cardiovascular effects (16), less is known regarding the mechanisms behind the biosynthesis of methylarginines. The aim of the present study was to examine whether the degradation of endogenous protein via the proteasome and autophagy pathways affects the production of methylarginines.

\section{Materials and methods}

Chemicals and reagents. L-Arg, MMA, ADMA, SDMA, $N$-propyl-L-arginine (N-PLA) and chloroquine were purchased from Sigma-Aldrich (St. Louis, MO, USA). Cbz-L-Leu-L-Leu-L-Leu-al (MG132) and epoxomicin were from the Peptide Institute (Osaka, Japan). High-performance liquid chromatography (HPLC)-grade methanol and acetonitrile were from Wako (Tokyo, Japan). A Bond Elut SCX cartridge was obtained from Varian (Harbor City, CA, USA). The AccQ-Tag Ultra-Fluor ${ }^{\mathrm{TM}}$ derivatization kit (borate buffer 
and reagent) was obtained from Waters (Milford, MA, USA). Angiotensin II (Ang II) and bradykinin 1-7, calibration standards for MALDI-QIT-TOF/MS, were purchased from Sigma-Aldrich. 2,5-Dihydroxybenzoic acid (DHBA) matrix of MALDI-MS grade was obtained from Shimadzu GLC (Tokyo, Japan).

Cell culture. The human embryonic kidney 293T (HEK293T) and lung epithelial A549 cell lines were maintained in Dulbecco's Modified Eagle's Medium (DMEM; Nissui, Tokyo, Japan) supplemented with $10 \%$ heat-inactivated fetal bovine serum (Life Technologies, Carlsbad, CA, USA) at $37^{\circ} \mathrm{C}$ in a humidified atmosphere containing $5 \% \mathrm{CO}_{2}$.

Sample preparations. HEK293T and A549 cells were separately plated at a density of $1 \times 10^{7}$ cells. After $48 \mathrm{~h}$, the cells were treated with proteasome or autophagy inhibitors and harvested in PBS. The supernatants were removed by centrifugation at $1,000 \mathrm{x}$ g for $2 \mathrm{~min}$, and the precipitated cells were re-suspended in ice-cold Milli- $\mathrm{Q}^{\mathrm{TM}}$ water. After boiling for $10 \mathrm{~min}$, the samples were incubated for $5 \mathrm{~min}$ at room temperature and then maintained on ice for $10 \mathrm{~min}$. Cell debris and insoluble matter were removed by centrifugation at 16,000 $\mathrm{x} \mathrm{g}$ for $10 \mathrm{~min}$. Protein concentrations in the lysates were determined using Bio-Rad protein assay reagent (Bio-Rad, Hercules, CA, USA), and the cell lysates were divided into aliquots of $1.8 \mathrm{ml}$. Each aliquot was acidified with $200 \mu \mathrm{l}$ of $10 \%$ 5-sulfosalicylic acid and dissolved in Milli-Q water to a total volume of $2.0 \mathrm{ml}$. Samples were vortexed, maintained on ice for $10 \mathrm{~min}$ for complete protein precipitation, and centrifuged at $16,000 \mathrm{x}$ g for $10 \mathrm{~min}$. The supernatant was reserved and maintained on ice until solid-phase extraction (SPE).

SPE and derivatization. Deproteinized samples were subjected to crude fractionation on a Bond Elut SCX cation exchange cartridge as previously reported (17). After final elutions of SPE, eluted basic molecules were dried with Savant SpeedVac ${ }^{\text {TM }}$ concentrator (Thermo Fisher Scientific, Waltham, MA, USA). Amino acid derivatization with AccQ-Tag reagents was conducted as previously described (17). Briefly, $25 \mu \mathrm{l}$ of either a standard mix solution or cell extract was combined with $25 \mu \mathrm{l}$ of AccQ-Tag Ultra borate buffer, then $10 \mu \mathrm{l}$ of AccQ-Tag reagent previously dissolved in $1.0 \mathrm{ml}$ of AccQ-Tag Ultra reagent diluent was added. The reaction was allowed to proceed for $10 \mathrm{~min}$ at room temperature.

Antibodies and Western blotting. Monoclonal antibodies against ubiquitin (P4D1) and $\beta$-actin were purchased from Santa Cruz Biotechnology (Santa Cruz, CA, USA) and Sigma-Aldrich, respectively. Anti-microtubule-associated protein 1 light chain 3 (MAP1LC3) polyclonal antibody was from MBL International (Woburn, MA, USA). Western blot analysis was performed as previously described (18).

Chromatographic conditions. Ultra high-performance liquid chromatography (UPLC) was performed on an Acquity ${ }^{\mathrm{TM}}$ UPLC system (Waters) equipped with fluorescence detection. 6-Aminoquinolyl carbamoyl (AQC) derivatives were separated on an AccQ-Tag ${ }^{\text {TM }}$ amino acid analyzer $\mathrm{C}_{18}$ column (Waters, $1.7 \mu \mathrm{m}, 2.1 \times 100 \mathrm{~mm}$ ) with a VanGuard ${ }^{\mathrm{TM}}$ cartridge (Waters) at $40^{\circ} \mathrm{C}$ with an acetonitrile gradient in ammonium acetate buffer (pH 6.0) at a flow rate of $0.25 \mathrm{ml} / \mathrm{min}$. Mobile phase solutions A and B were made up of 4 and $30 \%$ acetonitrile, respectively, in $0.1 \mathrm{M}$ sodium acetate $\mathrm{pH}$ 6.0. The gradient conditions were $\mathrm{A}: \mathrm{B}=100: 0$ for $25 \mathrm{~min}$, then $93: 7$ for $>45 \mathrm{~min}$, hold at $93: 7$ for $75 \mathrm{~min}$, flushing with 0:100 for $5 \mathrm{~min}$, then a return to initial conditions of 100:0 for $>1 \mathrm{~min}$. The column was allowed to re-equilibrate for $10 \mathrm{~min}$. The injection volume was $7.5 \mu 1$.

For the assessment of purity, the fractionated peaks were re-chromatographed by HPLC (Alliance 2695 separation module and 414 fluorescence detector, Waters). Chromatographic separation was achieved on a Cosmosil

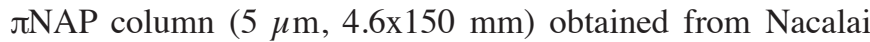
Tesque (Kyoto, Japan). The column was operated at $30^{\circ} \mathrm{C}$. After sample injection $(100 \mu \mathrm{l})$, separation was performed under isocratic conditions with $25 \%$ methanol as a solvent, and maintained for $45 \mathrm{~min}$. In order to elute strongly bound compounds, the column was flushed with $100 \%$ methanol for $5 \mathrm{~min}$ and re-equilibrated under isocratic conditions for $10 \mathrm{~min}$ prior to the next injection. In both the UPLC and HPLC systems, fluorescence from the analytes was detected at $\lambda_{\text {ex }} 250 \mathrm{~nm}$ and $\lambda_{\text {em }} 395 \mathrm{~nm}$. Data were collected using Empower $^{\mathrm{TM}} 2$ HPLC software for Windows (Waters).

MALDI-QIT-TOF/MS. DHBA matrix $(10 \mathrm{mg})$ was dissolved in $1 \mathrm{ml}$ of $40 \%$ acetonitrile and $0.1 \%$ trifluoroacetic acid. Matrix solution $(0.5 \mu \mathrm{l})$ and $1 \%$ sample $(0.5 \mu \mathrm{l})$ dissolved in methanol and water were deposited on a MALDI plate and left to dry at room temperature for the preparation of sample spots. External calibrations were achieved using the standard reagents Ang II [monoisotopic mass $(\mathrm{M}+\mathrm{H})^{+}=1046.5$ ], and bradykinin 1-7 $\left[(\mathrm{M}+\mathrm{H})^{+}=757.4\right]$. Tandem mass experiments with MALDI-QIT-TOF/MS ${ }^{\mathrm{n}}(\mathrm{n}=1,2$, or 3) were performed using argon gas as the collision gas on an AXIMA-QIT mass spectrometer (Shimadzu, Kyoto, Japan) equipped with a nitrogen laser $(337 \mathrm{~nm})$ with the collision-induced dissociation control value set at 160 (19).

Standard curves. Calibration standards for the methylarginine derivatives $(0,6.25,12.5$ and $25.0 \mathrm{ng})$ were prepared from stock solutions $(0.4 \mathrm{mg} / \mathrm{ml}$ in $0.1 \mathrm{M} \mathrm{HCl})$ diluted in Milli-Q water and stored at $-20^{\circ} \mathrm{C}$. For the preparation of the calibration curves, the cell lysates were spiked with standards for arginine derivatives and an internal standard (200 ng N-PLA) and subjected to SPE, derivatization and chromatography as described above. Calibration curves were calculated by plotting the ratio of the peak area of analytes to the area of the internal standard (N-PLA) vs. analyte quantity. For the determination of the recovery rate, standard solutions (25 ng of MMA, ADMA and SDMA) were subjected to SPE, derivatization and chromatographic separation.

Statistical analysis. Data are presented as the mean weight \pm standard error. Statistical analysis was performed using the Student's t-test.

\section{Results}

Development and validation of methods. Fig. 1A shows typical UPLC (equipped with a $\mathrm{C}_{18}$ column) chromatograms obtained 
A

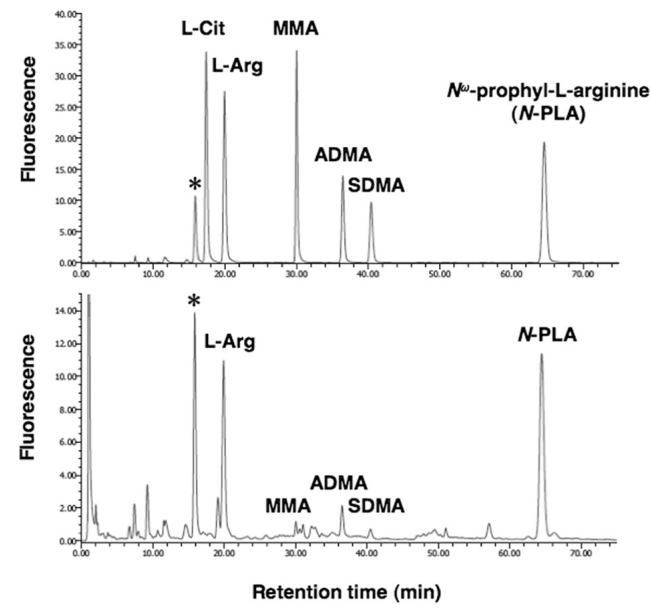

C

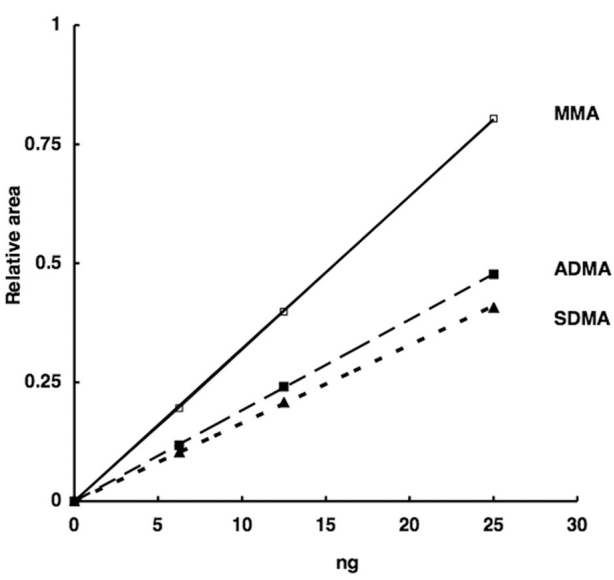

B

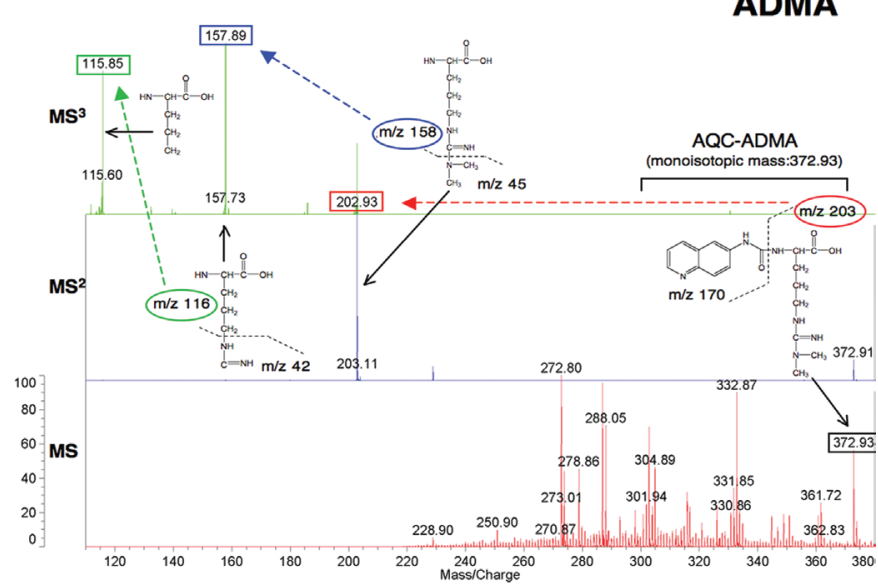

SDMA

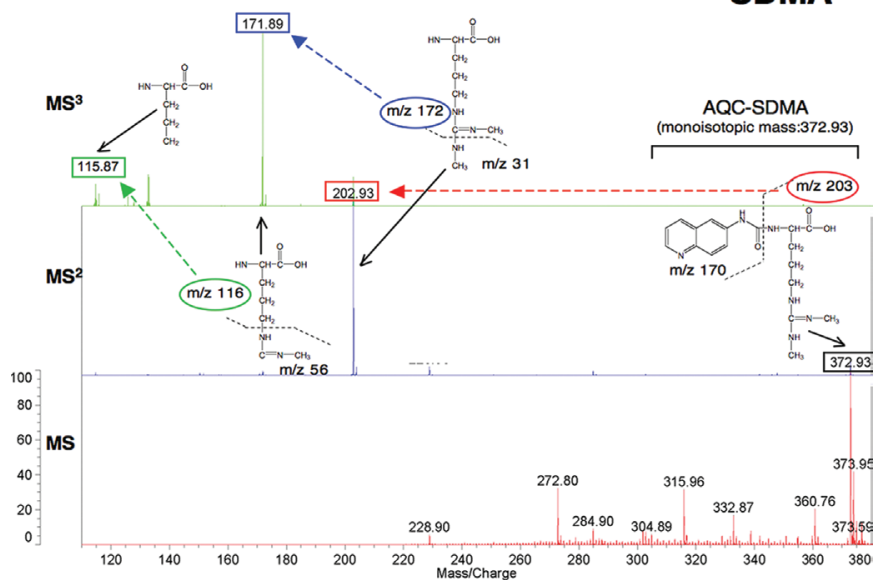

Figure 1. Qualification and quantification of methylarginines. (A) Representative UPLC chromatogram of a combined standard containing $13.6 \mathrm{ng}$ L-Arg, L-citrulline (L-Cit), MMA, ADMA, SDMA and N-PLA (upper panel). Typical chromatogram of a cell lysate obtained from HEK293 cells spiked with the internal standard N-PLA (lower panel). Chromatographic conditions were as described in the Materials and methods. "By-product interference (AMQ; 6-aminoquinoline). (B) MALDI-QIT-TOF/MS spectra fragmentation pattern of the AQC-derivatized ADMA (upper panel) and SDMA (lower panel) isomers and their related structures. Arrows, specific fragment ion for each metabolite; numbers in ovals, theoretical molecular masses (m/z) in the structural formula; numbers in boxes, experimental values; dashed arrows, transition of molecular mass (m/z). (C) Standard curves for the ratio of peak area to weight for MMA, ADMA and SDMA. Conditions were as in (A).

after injection of a standard sample containing methylarginine derivatives (upper panel) and samples of HEK293T cell lysate (lower panel). The retention times of each arginine derivative were as follows: L-Arg, $20.1 \mathrm{~min}$; MMA, $29.9 \mathrm{~min}$; ADMA, $36.7 \mathrm{~min}$; SDMA, $40.7 \mathrm{~min}$; and N-PLA, $64.5 \mathrm{~min}$. The peak at $15.9 \mathrm{~min}$ was due to by-product interference (6-aminoquinoline; AMQ). Peaks were fractionated corresponding to ADMA and SDMA in the cell lysates, and their purity was confirmed by HPLC re-chromatography with the $\pi$ NAP column, which has a different separation mechanism than the $\mathrm{C}_{18}$ column used previously.

$M S, M S / M S$ and $M S / M S / M S$. The identities of the collected dimethylarginines were assessed using MALDI-QIT-TOF/MS . Representative fragmentation patterns of AQC-derivatized ADMA $\left[(\mathrm{M}+\mathrm{H})^{+}=372.9\right]$ and SDMA $\left[(\mathrm{M}+\mathrm{H})^{+}=372.9\right]$ from A549 cells are shown in Fig. 1B. Since ADMA and SDMA are isomers, a shared transition of $372.9 \rightarrow 202.9$ (which results in the elimination of AQC group) produced the most abundant fragment ion common to both. Structure-dependent specific daughter transitions for the individual isomers were found to be $202.9 \rightarrow 157.9$ for $\mathrm{m} / \mathrm{z}$ ADMA and $202.9 \rightarrow 171.9$ for SDMA (Fig. 1B).

Recovery and calibration. The recovery rates of all the arginine derivatives were as follows: MMA, $87.8 \% \pm 6.2$, ADMA, $93.9 \% \pm 1.1 \%$; SDMA, $81.3 \% \pm 2.3 \%$. The linearities were verified over the assay range (0-25 ng on-column). Calibration curves were calculated using the peak area vs. the concentrations of the standard analytes. Typical linear regression equations from the standard curves of each analyte were: MMA, $y=1.237 \times 10^{5} x$; ADMA, $y=1.237 \times 10^{5} x$; and SDMA, $y=9.97 \times 10^{4} x$, where $y$ is the peak area ratio of the analyte to the internal standard and $x$ is the analyte amount (ng). The correlation coefficients for the MMA, ADMA and SDMA standards in the cell lysates were $0.999,1.0$ and 0.9998 , respectively.

Effect of proteasome inhibition on cellular concentrations of methylarginines. The inhibition of proteasome function prevents the rapid degradation of ubiquitinated proteins. A 4-h 
A

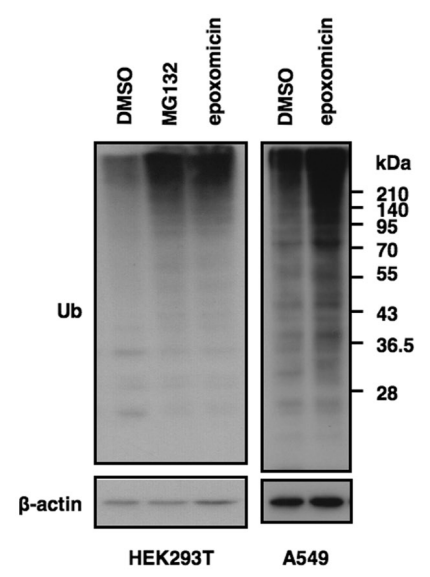

B

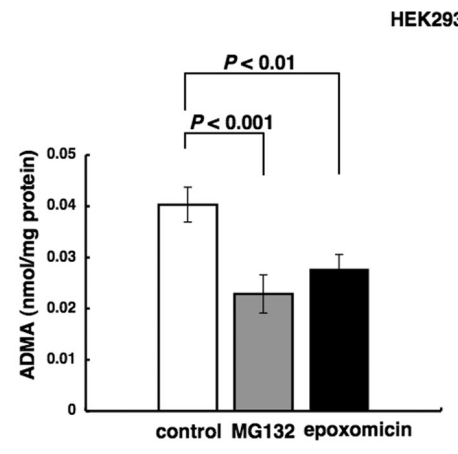

C

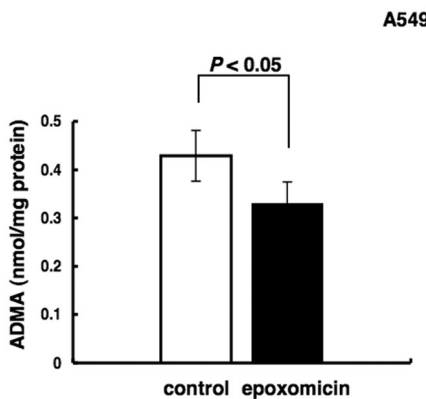

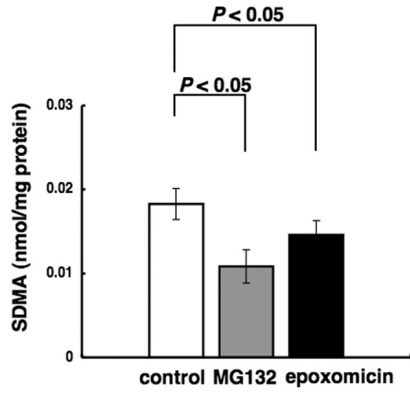

549

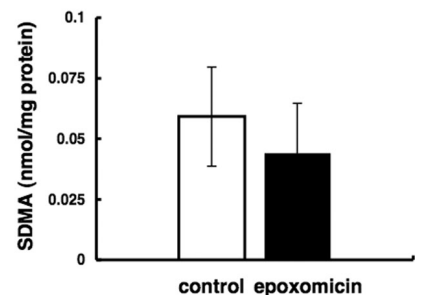

Figure 2. Effect of proteasome inhibition on methylarginine production in cells. (A) MG132 (10 $\mu \mathrm{M})$ or epoxomicin (0.5 $\mu \mathrm{M})$ leads to the accumulation of ubiquitin-conjugated proteins in HEK293 (left panel) and A549 (right panel) cells. Cellular concentrations of ADMA and SDMA in (B) the cultured HEK293 cells with or without MG132 or epoxomicin, and (C) the cultured A549 cells with or without epoxomicin. Data are presented as the mean \pm SD of triplicate determinations in $\mathrm{nmol} / \mathrm{mg}$ of protein. $\mathrm{P}<0.05, \mathrm{P}<0.01$ and $\mathrm{P}<0.001$ vs. the control group (DMSO), determined by the Student's t-test.

A

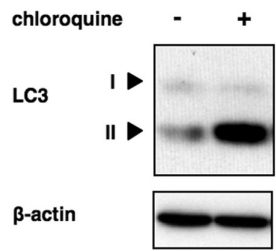

B

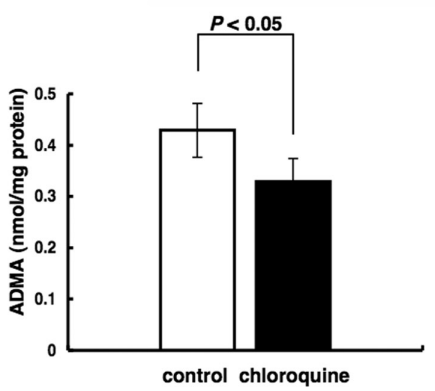

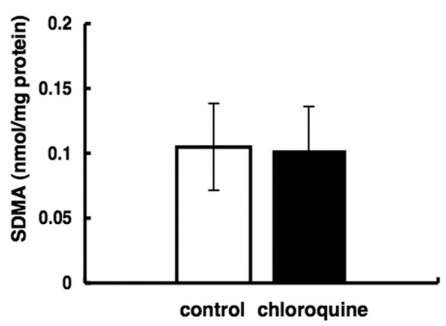

Figure 3. Effect of autophagy inhibition on methylarginine production in cells. (A) Chloroquine (50 $\mu \mathrm{M})$ leads to the accumulation of LC3-II in A549 cells. (B) Cellular concentrations of ADMA and SDMA in the cultured A549 cells with or without chloroquine. Data are presented as the mean \pm SD in $\mathrm{nmol} / \mathrm{mg}$ of protein. $\mathrm{P}<0.05$ vs. the control group (water), determined by the Student's t-test.

incubation of HEK293 cells with MG132 (10 $\mu \mathrm{M})$ and epoxomicin $(0.5 \mu \mathrm{M})$ or A549 cells with epoxomicin $(0.5 \mu \mathrm{M})$ caused the accumulation of ubiquitin-conjugated proteins, as determined by Western blotting using anti-ubiquitin antibodies (Fig. 2A). Under these conditions, the cellular concentration of ADMA and SDMA in the HEK293 cells was decreased by $30-40 \%$ compared to the untreated cells (Fig. 2B). While the treatment of A549 cells with epoxomicin reduced the level of ADMA by $\sim 40 \%$ compared to untreated cells, SDMA was not significantly altered (Fig. 2C).

Effect of autophagy inhibition on cellular concentrations of methylarginines. MAP1-LC3 has been reported to be a useful marker protein for the determination of mammalian autophagy. After its translation, the precursor protein is processed at the C-terminus, becoming an $18 \mathrm{kDa}$ protein (LC3-I). LC3-I conjugates with phosphatidylethanolamine and is transformed into a membrane-bound mature form (LC3-II) according to the formation of autophagosomes (20). As shown in Fig. 3A, the amount of LC3-II in the A549 cells was increased following $12 \mathrm{~h}$ of treatment with $50 \mu \mathrm{M}$ chloroquine. This suggests that chloroquine caused the suppression of autophagy. Under this condition, the levels of ADMA, but not SDMA, were found to be significantly decreased (Fig. 3B).

\section{Discussion}

The measurement of cellular methylarginine derivatives is challenging due to their relatively low amounts, difficulty in chromatographic separation, and the lack of simple and reliable detection methods (21). In the present study, to overcome these obstacles, a sensitive and accurate quantification method 
was established using UPLC with high resolution between the ADMA and SDMA isomers (Fig. 1A) combined with the AccQ-fluor labeling system. For the labeling of methylarginines, the AccQ-fluor method is simpler than techniques using fluorescent reagents such as ortho-phthaldialdehyde, and allows for a shorter reaction time (17). MMA, ADMA and SDMA are well separated, and N-PLA is sufficiently resolved to allow accurate integration of the internal standard peak. Other basic amino acids, such as citrulline, do not interfere with this assay (Fig. 1A). Since a $\pi-\pi$ interaction between the naphthyl group of the $\pi$ NAP column and the AQC group of the amino acids is expected, the $\pi$ NAP column exhibits a higher selectivity than the $\mathrm{C}_{18}$ column conventionally used for the separation of AQC derivatives. Using this column, the purity of the peaks was confirmed by HPLC. The structural identities of the HPLC-fractionated peaks corresponding to ADMA and SDMA were confirmed by MS, MS/MS, and MS/MS/MS sequential analyses with MALDI-QIT-TOF/ MS (Fig. 1B) (19). The present methods resulted in fairly good recovery rates $(>80 \%)$, and linear regression analysis showed significant linearity of the calibration curve (Fig. 1C).

While MMA and ADMA are known to be potent inhibitors of constitutive and inducible nitric oxide synthase (NOS), SDMA is inactive. NO, which is synthesized from L-Arg by NOS, plays multiple roles in the cardiovascular system, the immune system, and as a neurotransmitter in the brain. Therefore, MMA and ADMA are responsible for the regulation of NOS in vivo. It has been suggested that these free methylarginines are generated by the proteolysis of methylated proteins, but the underlying mechanisms of the protein arginine methylation have not been elucidated. Bulau et al (22) recently demonstrated that the proteasome inhibitor MG132 suppressed the production of ADMA, but not SDMA, in A549 cells. The present study examined whether endogenous protein degradation levels reflect the cellular amount of the methylarginine species. Treatment of HEK293T cells with the specific proteasome inhibitors MG132 or epoxomicin effectively suppressed protein degradation, and levels of free ADMA and SDMA were reduced (Fig. 2B). A significant decrease in ADMA, but not SDMA, was also observed in A549 cells treated with epoxomicin (Fig. 2C). We also examined whether autophagy is involved in the biogenesis of methylarginine using chloroquine. Chloroquine is thought to inhibit protein degradation by a dual mechanism: the neutralizing of lysosomes as it is a weak base (23), and the accumulation of larger autophagic vesicles (24). Since HEK293T cells were less sensitive to the inhibition of autophagy than A549 cells (data not shown), this study confirmed that the incubation of A549 cells with chloroquine significantly augmented the accumulation of LC3-II (Fig. 3A). While a significant decrease in cellular ADMA was observed, there was no change in SDMA levels in the A549 cells (Fig. 3B). These results suggest that both the proteasome and autophagy pathways are essential for the biosynthesis of methylarginines.

It remains to be determined whether the formation efficiency of the methylarginines is dependant on the cellular activity of PRMTs. Among the methylarginine derivatives, the role of MMA and ADMA in cardiovascular and kidney diseases or their associated complications as a consequence of endothelial dysfunction is of particular interest. Molecular approaches including the regulation of PRMTs may not only elucidate the mechanisms of methylarginine production, but may also to contribute to an understanding of the clinical implication of various diseases, including vascular disorders.

\section{Acknowledgements}

This work was supported in part by a Grant-in-Aid for Scientific Research (S) from the Ministry of Education, Culture, Sports, Science, Sports and Technology of Japan, and the TARA Project, University of Tsukuba.

\section{References}

1. Bedford MT and Clarke SG: Protein arginine methylation in mammals: who, what, and why. Mol Cell 33: 1-13, 2010.

2. Wolf S: The protein arginine methyltransferase family: an update about function, new perspectives and the physiological role in humans. Cell Mol Life Sci 66: 2109-2121, 2009.

3. Lin WJ, Gary JD, Yang MC, Clarke S and Herschman HR: The mammalian immediate-early TIS21 protein and the leukemiaassociated BTG1 protein interact with a protein-arginine N-methyltransferase. J Biol Chem 271: 15034-15044, 1996.

4. Lakowski TM and Frankel A: Kinetic analysis of human protein arginine N-methyltransferase 2: formation of monomethyl- and asymmetric dimethyl-arginine residues on histone $\mathrm{H} 4$. Biochem $\mathbf{J}$ 421: 253-261, 2009.

5. Tang J, Gary JD, Clarke S and Herschman HR: PRMT 3, a type I protein arginine N-methyltransferase that differs from PRMT1 in its oligomerization, subcellular localization, substrate specificity, and regulation. J Biol Chem 273: 16935-16945, 1998.

6. Chen D, Ma H, Hong H, et al: Regulation of transcription by a protein methyltransferase. Science 284: 2174-2177, 1999.

7. Frankel A, Yadav N, Lee J, Branscombe TL, Clarke S and Bedford MT: The novel human protein arginine $\mathrm{N}$-methyltransferase PRMT6 is a nuclear enzyme displaying unique substrate specificity. J Biol Chem 277: 3537-3543, 2002.

8. Lee J, Sayegh J, Daniel J, Clarke S and Bedford MT: PRMT8, a new membrane-bound tissue-specific member of the protein arginine methyltransferase family. J Biol Chem 280: 32890-32896, 2005.

9. Kim J-D, Kako K, Kakiuchi M, Park GG and Fukamizu A: EWS is a substrate of type I protein arginine methyltransferase, PRMT8. Int J Mol Med 22: 309-315, 2008.

10. Pollack BP, Kotenko SV, He W, Izotova LS, Barnoski BL and Pestka S: The human homologue of the yeast proteins Skb1 and Hs17p interacts with Jak kinases and contains protein methyltransferase activity. J Biol Chem 274: 31531-31542, 1999.

11. Miranda TB, Miranda M, Frankel A and Clarke S: PRMT7 is a member of the protein arginine methyltransferase family with a distinct substrate specificity. J Biol Chem 279: 22902-22907, 2004.

12. Cook JR, Lee J-H, Yang Z-H, Krause CD, Herth N, Hoffmann R, and Pestka S: FBXO11/PRMT9, a new protein arginine methyltransferase, symmetrically dimethylates arginine residues. Biochem Biophys Res Commun 342: 472-481, 2006.

13. Miyake $M$ and Kakimoto $Y$ : Synthesis and degradation of methylated proteins of mouse organs: correlation with protein synthesis and degradation. Metab Clin Exp 25: 885-896, 1976.

14. Drapier JC and Hibbs JB: Murine cytotoxic activated macrophages inhibit aconitase in tumor cells. Inhibition involves the iron-sulfur prosthetic group and is reversible. J Clin Invest 78: 790-797, 1986.

15. Vallance P, Leone A, Calver A, Collier J and Moncada S: Accumulation of an endogenous inhibitor of nitric oxide synthesis in chronic renal failure. Lancet 339: 572-575, 1992.

16. Calver A, Collier J, Leone A, Moncada S and Vallance P: Effect of local intra-arterial asymmetric dimethylarginine (ADMA) on the forearm arteriolar bed of healthy volunteers. J Hum Hypertens 7: 193-194, 1993.

17. Heresztyn T, Worthley MI and Horowitz JD: Determination of L-arginine and $N^{\mathrm{G}}, N^{\mathrm{G}}$ - and $N^{\mathrm{G}}, N^{\mathrm{G}}$-dimethyl-L-arginine in plasma by liquid chromatography as AccQ-Fluor fluorescent derivatives. J Chromatogr B Analyt Technol Biomed Life Sci 805: 325-329, 2004. 
18. Yamagata K, Daitoku H, Takahashi Y, et al: Arginine methylation of FOXO transcription factors inhibits their phosphorylation by Akt. Mol Cell 32: 221-231, 2008.

19. Kasai H, Tsubuki M, Shimada K, Nambara T and Honda T: Analyses of biologically active steroids: antitumor active OSW-1 and cardiotonic marinobufotoxin, by matrix-assisted laser desorption/ionization quadrupole ion trap time-of-flight tandem mass spectrometry. Chem Pharm Bull 57: 948-956, 2009.

20. Kabeya Y, Mizushima N, Ueno T, et al: LC3, a mammalian homologue of yeast Apg8p, is localized in autophagosome membranes after processing. EMBO J 19: 5720-5728, 2000.

21. Teerlink T: HPLC analysis of ADMA and other methylated L-arginine analogs in biological fluids. J Chromatogr B Analyt Technol Biomed Life Sci 851: 21-29, 2007.
22. Bulau P, Zakrzewicz D, Kitowska K, Wardega B, Kreuder J and Eickelberg O: Quantitative assessment of arginine methylation in free versus protein-incorporated amino acids in vitro and in vivo using protein hydrolysis and high-performance liquid chromatography. Biotechniques 40: 305-310, 2006.

23. Wibo M and Poole B: Protein degradation in cultured cells. II. The uptake of chloroquine by rat fibroblasts and the inhibition of cellular protein degradation and cathepsin B1. J Cell Biol 63: 430-440, 1974

24. Gray RH, Sokol M, Brabec RK and Brabec MJ: Characterization of chloroquine-induced autophagic vacuoles isolated from rat liver. Exp Mol Pathol 34: 72-86, 1981. 